Results The most common reasons for patients not accepting a CBT referral were referral to the Trust's inpatient neuropsychiatry ward, or non-attendance at assessment or treatment sessions. We identified a total of 98 patients with mFND and 76 ONP patients treated with CBT. $56.1 \%$ of $\mathrm{mFND}$ and $56.6 \%$ of ONP patients attended all sessions offered. There were no socio-demographic characteristics associated with mFND patients who completed all therapy sessions versus those who dropped out early. Compared to controls, mFND patients were more likely to be female $\left(72.4 \% \mathrm{v} .44 .7 \%, \chi^{2}\right.$ : 13.6, 95\% CI: $12.2-41.9, \mathrm{p}=0.001)$, unemployed $(52.6 \%$ versus $35.5 \%, \chi 2: 5,95 \% \mathrm{CI}: 2.2-30.8, \mathrm{p}=0.03)$, to have a carer $(27.6 \%$ versus $14.3 \%, \chi 2: 4.4,95 \%$ CI: 0.9-24.7, $\mathrm{p}=0.04)$ and to have experienced child sexual abuse $(23.8 \% \mathrm{v}$ $\left.8.2 \%, \chi^{2}: 7.3, p=0.01\right)$. The most common mFND symptom was weakness $(26.9 \%)$, most frequently in the leg or entire body. Both mFND and ONP patients showed significant improvements in measures of psychological functioning (measured with the CORE-OM, HoNOS-ABI, and PHQ-9), with physical symptoms improving in $49.4 \%$ of mFND patients. A logistic regression showed acceptance of psychological formulations prior to CBT $(\mathrm{p}<0.02)$ was associated with the improvement of physical symptoms in mFND patients.

Conclusions mFND patients treated in a specialist CBT clinic show similar improvements in physical and psychological functioning to ONP patients with positive effects on motor symptoms, distress, depression, general health and social functioning. With little evidence on the effectiveness of CBT in treating motor variants of FND, this study could help inform the design of a pilot RCT.

\section{8 \\ ARE THERE ANY FACTORS THAT PREDICT THE DIAGNOSIS OF EPILEPSY OR PSYCHOGENIC NON-EPILEPTIC SEIZURES (PNES) IN PATIENTS ADMITTED TO A SPECIALIST EPILEPSY UNIT?} ${ }^{1} \mathrm{~S}$ Gibson*, ${ }^{2} \mathrm{~S}$ Leighton, ${ }^{1,2} \mathrm{M}$ Oto. ${ }^{1} \mathrm{NHS}$ Greater Glasgow and Clyde; ${ }^{2}$ William Quarrier
Scottish Epilepsy Centre

\subsection{6/jnnp-2019-BNPA.38}

Introduction Differentiating between epilepsy and psychogenic non-epileptic seizures (PNES) can be difficult. Although clearly not a substitute for taking a careful history, certain patient characteristics may assist the clinicians towards diagnosis. The population of patients referred to an epilepsy specialist centre represent a complex and distinct group of patients and it is not clear which factors, if any, could point towards a diagnosis of epilepsy or PNES.

Aims/Objectives To investigate if the diagnosis of epilepsy or PNES is predicted by baseline demographic and clinical variables, including Hospital Anxiety and Depression (HADS) scores and medication prescription, in patients admitted to a specialist adult epilepsy centre.

Methods We conducted an observational retrospective cohort of consecutive patients admitted to the William Quarrier Scottish Epilepsy Centre (WQSEC) over a period of one year (01/ 09/16-01/09/17). Chosen predictor variables at baseline included: sex, age, employment education or training (EET), Scottish Index of Multiple Deprivation 2016 (SIMD 2016) rank status, attack frequency, length of index admission, number of anti-epileptic agents prescribed, prescription of benzodiazepines, of analgesia, or of psychotropic medications, and
HADS scores. Outcome measures were diagnosis of epilepsy or PNES, from diagnosis made by expert clinicians on discharge from index admission. Because of the presence of dual diagnosis, two multivariable binary logistic regression models were built - one for the epilepsy and one for the PNES diagnosis outcomes.

Results 50/73 (69\%) of patients admitted were diagnosed with epilepsy and 39/73 (53\%) with PNES. These respective groups include 16/73 (22\%) who had a dual diagnosis of both epilepsy and PNES. The model to predict epilepsy showed that significant individual predictor variables included number of antiepileptic agents prescribed (Odds Ratio (OR) $=3.59 \quad(95 \%$ CI: $1.37,9.42), p=0.010)$, prescription of psychotropic medications $(\mathrm{OR}=0.19(95 \% \mathrm{CI}: 0.04,0.91), \mathrm{p}=0.038)$, and length of index admission $(\mathrm{OR}=0.89 \quad(95 \% \mathrm{CI}$ : $0.81,0.98)$, $\mathrm{p}=0.018$ ). The model to predict presence of PNES revealed only one significant individual predictor variable, which was EET status (OR 0.13 (95\%CI: 0.02, 0.86), p=0.035).

Conclusions Baseline clinical and demographic factors may be of some utility to the clinician in anticipating a diagnosis of epilepsy or of PNES.

\section{THE IMMUNOMODULATORY EFFECT OF CLOZAPINE IN PATIENTS WITH TREATMENT RESISTANT SCHIZOPHRENIA: A RETROSPECTIVE COHORT STUDY}

${ }^{1}$ Lisshammar J, ${ }^{1}$ Blackman G, ${ }^{1}$ Carter B, 'Zafar R, ' Stewart R, ${ }^{1}$ Pritchard M, ${ }^{1}$ Pollak T, ${ }^{1}$ Rogers J, ${ }^{1}$ Cullen A, ${ }^{1}$ McGuire P, ${ }^{2}$ David A, ${ }^{1}$ MacCabe J. ${ }^{1}$ Institute of Psychiatry, Psychology and Neuroscience, King's College London, Dr Crespigny Park, London, UK.; ' Institute of Mental Health, University College London, London, UK

\subsection{6/jnnp-2019-BNPA.39}

Objectives The pathoetiology of Schizophrenia remains elusive, however, a growing body of literature suggests immune dysfunction may contribute. Clozapine, an atypical antipsychotic, has superior efficacy in treatment-resistant Schizophrenia compared to other antipsychotics - however underlying mechanisms remain unknown. Clozapine has recognised immunomodulatory effects, responsible for potentially fatal haematological side-effects - such as agranulocytosis. Whether Clozapine's immunomodulatory properties contribute toward its unique efficacy in treatment-resistant schizophrenia has not been systematically explored.

Methods A retrospective cohort study design was employed to examine the relationship between white cell, neutrophil, and platelet temporal trajectories and Clozapine response in treatment-resistant schizophrenia. Eligible patients were initiated on Clozapine for the first time and continued treatment for at least twelve weeks between 2007 and 2014 within the South London and Maudsley NHS Foundation Trust, and underwent weekly haematological monitoring. Retrospective clinical ratings were performed at baseline and three months following initiation, based upon patients' electronic clinical notes accessed through the Maudsley BRC Clinical Records Interactive Search system. Treatment response was defined as 'much' or 'very much' improved on the Clinical Global Impression Improvement subscale. Serial cell counts were extracted from a Clozapine haematological monitoring database.

Results Of 188 included patients, 114 (61\%) responded to treatment. Response did not significantly vary by ethnicity or age. Mean interval between haematological assessments was 6.9 (SD 2.0) days. General linear models revealed a significant increase from baseline for all cell lines in the second 